\title{
Groin Pain in the Athlete: Non-Bony Pathology Including Sportsman's Groin
}

\author{
Chris D. Mann and David M. Lloyd
}

Department of Surgery, University Hospitals of Leicester, Leicester Royal Infirmary, Leicester, UK

\begin{abstract}
Groin pain is a common complaint amongst sportsmen and women accounting for nearly $10 \%$ of all sports related injuries. This article focuses on non-bony causes of groin pain in sportsmen, particularly upon the condition commonly known as 'sportsman's hernia' or 'sportsman's groin'. It also highlights a new concept for the cause of groin pain in that the authors consider that many elite athletes with groin pain have pain arising from their inguinal ligament attachment. A new procedure known as the Lloyd Release Procedure has been specifically developed to treat this cause of groin pain. By performing a laparoscopic release of the inguinal ligament and reinforcing the groin with a mesh also placed laparoscopically, nearly all athletes are rendered pain free and most players can resume their activities within weeks. In a prospective study on over 140 elite athletes undergoing the Lloyd Release Procedure more than $90 \%$ of sportsmen returned to their normal sporting activities within four weeks of surgery.
\end{abstract}

Keywords: Groin pain, sportsman's groin, sportsman's hernia, inguinal ligament release, Lloyd release procedure.

\section{INTRODUCTION}

Groin pain is a common complaint amongst sportsmen and women, particularly those participating in sports involving kicking, rapid accelerations and deceleration, and sudden change of direction [1]. It has been estimated that groin injuries account for between $0.5-6.2 \%$ of sports-related injuries, with the prevalence in sports such as football as high as $5-15 \%$ [2,3]. Chronic groin pain can be difficult to diagnose, treat, and rehabilitate and is therefore responsible for a large proportion of time lost from sport and work in such athletes. This article focuses on non-bony causes of groin pain in athletes, particularly upon the condition commonly known as 'sportsman's hernia' or 'sportsman's groin'. The 'groin' comprises quite a large anatomical field from the lower abdomen and its muscles to the adductor muscle group insertion thereby encompassing the lower rectus abdominis muscle, the conjoined tendon, the superficial and deep inguinal rings, the tranvsversalis fascia, the inguinal and pectineal (Cooper's) ligaments, the pectineus muscle and fascia, adductor longus and brevis and even gracilis. Although many athletes are diagnosed with a so-called 'sportsman's hernia', careful clinical examination is important in these patients as most do not have a surgical 'lump' or hernia and they are more likely to have pain and tenderness of the ligamental attachments or tendinous insertions, particularly of the inguinal ligament, pectineal ligament and adductor tendon insertions. It must be remembered that all the insertions onto the pubic bone 'join together' and form an aponeurosis on the anterior aspect of the pubic bone (Fig. 1).

*Address correspondence to this author at the Department of Surgery, Leicester Royal Infirmary, Infirmary Square, Leicester, UK; Tel: $0044(0)$ 116265 3044; Fax: 0044 (0) 116265 3672; E-mail: dmlloyd1@aol.com

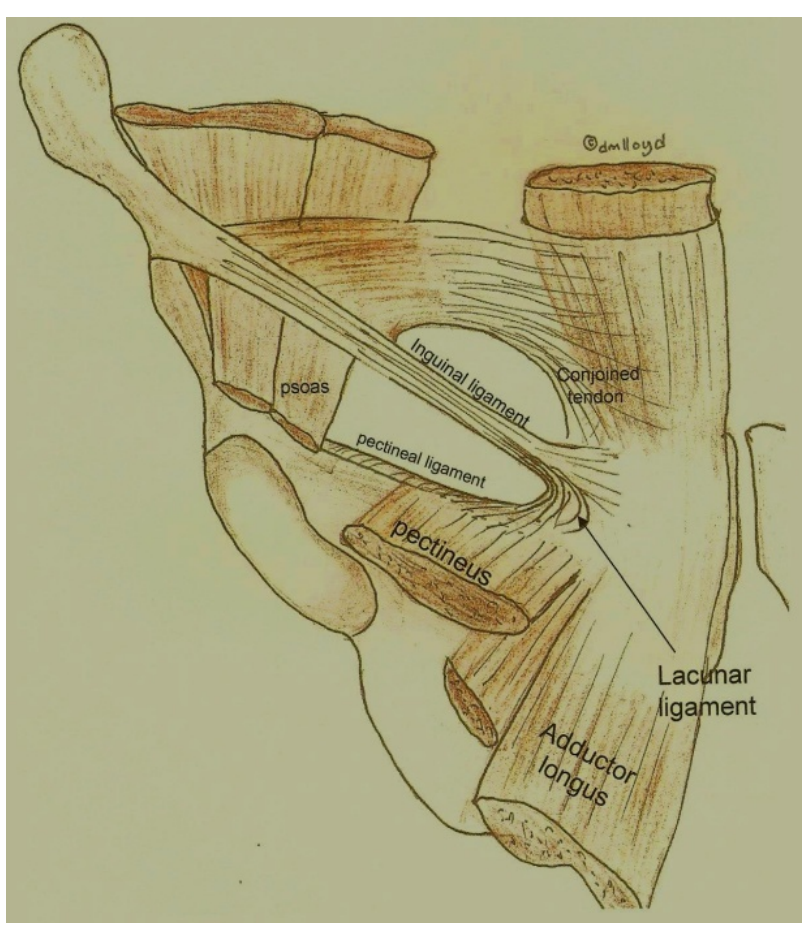

Fig. (1). Diagram showing ligaments, tendons and muscles around the pubic tubercle. There is an aponeurosis of the fibres of the rectus sheath, the inguinal ligament and the adductor tendon on the anterior part of the pubic bone.

Any part of this aponeurosis can become disrupted and cause groin pain. The aponeurosis is more pronounced in men, which may explain in part why groin pain is more common in males than in females. It is the authors' opinion that the term 'sportsman's hernia' should be abandoned as no formal hernia exists and the terms sportsman's groin or 
groin strain should be used. These terms better reflect the probable nature of the pathology. The tendons and ligaments exert tremendous forces at their attachments to the pubic bones which exert further pressures across the midline through the symphysis pubis and its fibrous cartilage. These stresses can be exacerbated with a number of conditions such as sacro-iliac mal-alignment, sacro-lumbar lower back strains and hamstring injuries. Correcting these additional conditions may improve the outcome of treatment for many causes of groin strains.

An extensive list of differential diagnoses exists for groin pain in athletes (Table 1). A careful clinical approach is required in these patients as description and localisation of the pain can be vague. In addition, the differential diagnosis should take into account a number of gastrointestinal, urological, gynaecological and testicular causes. Unfortunately, it should be recognised that a subset of these patients may have multiple pathology such that a single treatment or surgical procedure is unlikely to be curative. Alternative diagnoses should be considered in a child or adolescent, including septic arthritis, Perthe's disease, slipped upper femoral epiphysis and avascular necrosis of the hip. Chronic groin pain or inguinal pubalgia is generally divided into adductor disorders, osteitis pubis, sportsman's groin, and hip pathology. The principle non-bony causes of groin pain will be discussed below, along with nerve entrapment syndromes.

It is interesting that the majority of athletes do not attribute their symptoms to a single traumatic incident; nevertheless over $20 \%$ will definitely report an acute precipitating event or injury. Groin pain can be quite insidious and may evolve over weeks or months. Although many athletes have symptoms which stop their normal sporting activities at the time of physical exertion, more than half experience pain the following day, lasting for 24 hours or even 48 hours after strenuous exercise. Referred pain often confuses the clinical picture particularly as some patients with primary symptoms around the inguinal ligament may also have referred pain to the upper muscular belly of the adductor longus presumably via stimulation or entrapment of the obturator nerve. Secondary neuralgia, caused by stimulation of the ilio-inguinal, ilio-hypogastric, genito-femoral and/or obturator nerve and their branches must be considered in every patient. For ease of classification, the authors divide groin pain into two major groups. Those with upper groin pain - above and including the inguinal ligament, and those with definite lower groin pain which represents true adductor/gracilis tendon insertion - type pain. This differential based on the patients symptoms and clinical examination is important as surgery to the adductor region is, at best, unpredictable in its outcome, yet treatment options are far more successful in athletes with symptoms with upper groin pain around the inguinal region and pubic tubercle.

In athletes presenting with upper groin pain, the pain is often attributed to a dilated internal or even external inguinal ring. While there is no doubt that in the early stages of the development of an inguinal hernia patients experience pain at the deep ring, most athletes do not have tenderness at the deep ring. The pain is often more medial towards the pubic tubercle. Furthermore, the clinical assessment of the external ring is very subjective and any conclusions drawn with regard to this should be guarded. Palpation of the external ring by invaginating the scrotum often causes pain in normal subjects and may lead to an incorrect diagnosis of a sportsman's groin. Most normal males, who are non-athletes, will experience pain if someone tries to push the upper scrotum through the external ring! The 'invagination technique' can be very misleading. Whilst the authors are therefore very sceptical about this particular physical manouvre, there are some findings which may be helpful. Gentle palpation through the external ring allows the inguinal and lacunar ligaments to be palpated. If the athlete has unilateral symptoms direct comparison can be made with the contra-lateral inguinal ligament. Tenderness at this site may therefore confirm inguinal and lacunar ligament tenderness which can be quite striking in some athletes. These athletes may well have inguinal ligament strain or disruption, probably the most common cause of a sportsman's groin. The diameter of the external ring varies quite significantly in normal subjects and probably bears no correlation with the presence or absence of groin symptoms.

Pain above the inguinal ligament may be due to a posterior inguinal wall 'disruption' but this needs further comment. In the authors experience of performing more than six thousand laparoscopic hernia repairs, seldom were any 'tears' seen in the transversalis fascia. Moreover, a lax or bulging transversalis fascia within Hesselbach's triangle is often seen laparoscopically in older athletes over 40 years, but it is very debateable whether these findings contribute to any symptoms. Whilst plausible, as a cause of groin pain, posterior wall weaknesses are difficult to prove surgically. In contrast to a laparoscopic examination, examining the posterior wall through a small anterior wall crease incision can be limited and conclusions should only be drawn by experienced groin surgeons. The examination of these particular tissues is not an exact science as there are no normal reference values to which to relate. Small bulges can often be simply physiologically normal in many young men for example, yet they may still benefit from a surgical repair.

\section{CLASSIC SPORTSMAN'S GROIN - PAIN AROUND THE PUBIC TUBERCLE}

Sportsman's groin is a term used to describe a poorly defined, and under researched, syndrome of painful symptoms relating to the inguinal region around the inguinal ligament without a clinically recognisable hernia [4-6]. It is often termed 'inguinal-pubalgia'. The term sportsman's 'hernia', or Gilmore's groin, was first described by O.J. Gilmore in 1980 [2]. It is a syndrome characterized by chronic groin pain allegedly associated with a dilated superficial inguinal ring, although the exact cause of this injury is largely speculative and could possibly be multifactorial. It is the authors' opinion that the term 'sportsman's hernia' should therefore be abandoned as there is rarely a hernia present. The true incidence of sportsman's groin remains controversial, largely due to inconsistencies in definitions throughout the literature; some authors believe it is only a rare cause of groin pain in athletes, but the authors and others believe it is the most common cause of chronic groin pain and largely relates to the inguinal/pectineal attachments. Various explanations exist for this disorder, including avulsion of the conjoined tendon from the pubic 
Table 1. Differential Diagnosis

- Sportsman's groin

- Hernia -

Inguinal, femoral, obturator

- $\quad$ Nerve entrapments -

Ilioinguinal, iliohypogastric, obturator, genitofemoral, lateral femoral cutaneous

- $\quad$ Adductor tendinitis

- $\quad$ Muscle strains -

Rectus femoris, Rectus abdominis, Adductors, Sartorius

- Osteitis pubis

- Bursitis

- Hip disorders -

Osteoarthritis, Perthes disease, Synovitis, slipped capital femoral epiphysis, osteochondritis desiccans, Avascular necrosis of femoral head

- Lumbar spine disorders -

Osteoarthritis, intervertebral disc prolapse

- $\quad$ Fractures including stress and avulsion fractures -

Inferior pubic ramus, femoral neck, anterior superior iliac spine, anterior inferior iliac spine, Ischial tuberosity

- $\quad$ Lower Gastrointestinal disorders -

Diverticular disease, inflammatory bowel disease, appendicitis, mesenteric adenitis, malignancy

- $\quad$ Urological causes -

Ureteric calculi, urinary tract infections

- $\quad$ Gynaecological causes -

Pelvic inflammatory disease, endometriosis, pathology of ovaries and fallopian tubes

- $\quad$ Testicular causes -

Epididymo-orchitis, varicocoele, trauma, tumours

- Inguinal lymphadenopathy

tubercle, weakening of transversalis fascia, tears in internal oblique, tearing of external oblique or its aponeurosis causing superficial inguinal ring dilatation, and abnormalities of rectus abdominis insertion [4,5,7,8]. Several authors feel that a deficiency of the posterior inguinal wall is the most common operative finding in these patients yet it is hard to understand how this 'deficiency' can cause such intense pain. Furthermore, many athletes have an incidental weakness yet it is not the site of their symptoms.

It must also be remembered that the superior pubic ramus and pubic symphysis act as a compression strut linking the femur to the posterior pelvis and spine, with the centre of rotation near the pubic symphysis. Restricted hip movements, hip and abdominal strength in-coordination, imbalances in the pelvic biomechanics all serve to increase the stress through the pubic symphysis [9] which could exacerbate anterior groin pain. Abdominal and hip muscles act synergistically to control leg movement during sports. Weakness or poor coordination of these muscles may result in instability and overuse injury, particularly at the noncontractile structures of the inguinal region such as the transversalis fascia, rectus abdominis insertion, conjoined tendon, and external oblique aponeurosis [10]. The authors' personal opinion is that the pathology causing the majority of non-bony upper groin pain relates to an enthesopathy of one of the attachments of the ligaments around the pubic tubercle which may be analogous to tennis elbow, yet without significant degeneration of the attachment.

In many athletes, the pain tends to be of insidious onset, although some may experience a sudden tearing sensation which is exacerbated by exercise. Activities which increase intra-abdominal pressure may precipitate the pain, for example coughing and sneezing, although simple movements such as getting out of a car can often cause the pain also. There is no doubt that some athletes experience pain during exercise whilst others complain of pain and stiffness for 24 or 48 hours after strenuous activity. These delayed symptoms often cause the athlete to seek help. Although pain is felt in the groin, it may radiate into the adductor muscles, perineum or rectus muscle. The patient is typically male and in his twenties, however the syndrome has been described in females and a wide age range. Clinical examination often reveals tenderness around the pubic tubercle. In many patients, the inguinal ligament, palpated by scrotal invagination, is often exquisitely tender. In very slim athletes the inguinal ligament and lacunar ligaments can be palpated. It is the authors' belief that many athletes have pain caused by disruption of this 'inguino-pectineal' ligament complex. This complex involves the inguinal ligament, the curved fibres of the lacunar ligament, the pectineal ligament, the pectineus muscle, some posterior rectus sheath fibres, and some of the anterior fibres of the pubic bone aponeurosis. The patients who describe groin symptoms yet present with elusive clinical signs may well have disruption of the deep fibres of the inguinal and lacunar ligaments. There is usually no evidence of small bowel herniation through the deep or superficial rings and apart from a normal physiological cough impulse no hernia can be detected. It should be recognised that these patients may suffer from multiple synchronous pathologies. Imaging is mainly utilised to exclude differential diagnoses. Pelvic 
radiography may reveal changes of osteitis pubis (Fig. 2), stress fractures or skeletal disease, and can be supplemented by bone scans or CT scans as necessary. Flamingo view pelvic radiography can be used to demonstrate pelvic instability. MRI may be used to detect muscle strains, labral tears, osteitis pubis, ilio-psoas bursitis and stress fractures [11]. Ultrasound scanning enables dynamic assessment of the inguinal region and may demonstrate a increase in crosssectional area of the inguinal canal near the superficial ring upon straining, associated with a convex anterior bulge of the posterior canal wall, however is highly operatordependent [12].

\subsection{Conservative Management}

Conservative treatments generally have low success rates [13]. Conservative management involves drug treatment with non-steroidal anti-inflammatory drugs (NSAIDs), heat or ice, massage and physiotherapy. Transcutaneous electrical nerve stimulation (TENS) has also been used. Programmes involve initial rest of 6-8 weeks, followed by physiotherapy to improve coordination and progressively strengthen and stretch the hip muscles and core abdominal muscles. These exercises include resisted hip abduction, adduction, flexion and extension exercises, abdominal sit-ups, and slide-board exercises. Athletes then progress to sports-specific exercises and gradual return to full activities [13]. Return to competition occurs when the athlete is pain-free and training fully. Rehabilitation focuses on improving core strength, endurance and coordination, as well as resolving imbalances of the hip and abdominal muscles in order to stabilise the pelvic ring.

\subsection{Operative Management}

Conservative therapies may temporarily alleviate the patient's pain, but definitive surgical management is recommended should they fail. Although there are no studies which have demonstrated the optimal time to perform a surgical procedure, most clinicians would recommend waiting at least six weeks from the onset of symptoms. Operative treatment for chronic groin pain is therefore usually indicated if patients have had pain for 6 weeks or longer despite rest, NSAIDs and physiotherapy. The majority of operative interventions thus far for this condition have attempted to support the posterior wall of the inguinal canal, using a standard mesh hernia repair [14-18], a modified hernia repair [19], Bassini repair [20,21], or more recently a mesh placed laparoscopically either using an extra-peritoneal technique (TEP) [22-25] or a transabdominal pre-peritoneal (TAPP) technique [25-28].

\subsection{Conventional-Type Hernia Repairs}

The original operation described by Gilmore involved a 6-layer repair, plicating the transversalis fascia, re-attaching the internal oblique muscle to the pelvic bone, repairing the external oblique and tightening the external ring. Between 1980 and 2008, the Gilmore clinic performed over 4000 of these operations, reportedly with a $97 \%$ success rate [2]. The technique may have been recently modified by the additional use of a mesh. Brannigan et al. [19] reported a series of 85 athletes treated using a similar operation, demonstrating $96 \%$ returning to full competitive sport within 15 weeks of surgery. Several studies have since reported the use of open hernia repair, using either a modified Bassini repair $[4,20,21]$, or Lichteinstein mesh repair [16], with varying results. The authors prefer to use a laparoscopic method as laparoscopic hernia surgery has been shown to be associated with short hospital stay, improved post-operative pain, and improved convalescence [10,23-32]. It also has the theoretical advantage that the internal posterior position of the mesh behind the conjoined tendon and pubic bone should create a stronger repair than open surgery where the mesh is placed anteriorly. Several series have explored the benefits of laparoscopic hernia repair as a treatment modality in athletes with chronic groin pain [22-28]. Post-operative results range from $100 \%$ returning to sporting activities at 4 weeks, to $93 \%$ within 3 months. Genitsaris et al. reported a large retrospective series of 131 professional athletes who underwent bilateral TAPP for groin pain, with 97\% returning to sporting activity within 3 weeks [26]. Van Veen et al. reported a series of 55 athletes, 38 of whom were professional, who underwent laparoscopic TEP [22]. 88\% returned to sporting activity within 8 weeks, and $100 \%$ within 3 months. Paajanen et al. reported 41 professional athletes undergoing laparoscopic TEP, with 100\% having returned to sporting activity at 1 month [24]. Two of the smaller reported series report only professional athletes $[23,27]$. Overall, patients undergoing laparoscopic procedures for groin pain return to sports quicker than those undergoing open operations. There is therefore a significant amount of literature reporting the benefits of a laparoscopic approach.

\subsection{Minimal-Repair Technique}

Dr. Ulricke Muschaweck and colleagues from the Hernia Centre, Munich, Germany, have devised the 'minimal-repair technique' for sportsman groin pain. This is an open procedure performed under local anaesthetic and involves repairing the weak area of the posterior inguinal canal wall and reducing the tension of the attachment of the rectus abdominis into the pubic tubercle. It is understood that the procedure has been performed in several thousand athletes, including many high-profile elite athletes. It is claimed that athletes return to training at 2 days post operation with full recovery between 10 and 14 days. However as yet, this data has not been published and therefore formal conclusions cannot be drawn about its efficacy. Symptomatic relief in these patients may result from the sutures reinforcing the posterior wall of the inguinal canal, division of branches of the ilio-inguinal nerve and genito-femoral nerve, or alteration of the tension along the inguinal ligament.

\subsection{The Inguinal Ligament Release Procedure}

Despite the wide range of surgical procedures which have been developed for the treatment of groin strain, there remains a group of athletes who continue to complain of symptoms around the pubic tubercle. Furthermore, many players who have had an initial successful outcome with open surgical techniques unfortunately 'break down' and return with their original symptoms. In 1998, a Premier League football player who had undergone bilateral open hernia repair for groin strain was referred to Leicester with a recurrence of his groin pain. The player did not have a recurrent hernia but presented with severe pain at the attachment of the inguinal ligament on to the pubic tubercle. 
It was postulated that his pain was due to severe strain of the ligament and that releasing the ligament from the bone would improve his symptoms. He underwent a laparoscopic procedure where the inguinal ligament was divided and the internal aspect of the groin reinforced with a soft mesh. The operation was successful and he played and scored for this club 23 days after surgery. The release procedure for groin pain has been modified slightly over the past decade but it continues to be successful for the treatment of groin pain in elite athletes. The laparoscopic technique for the inguinal ligament release followed by mesh reinforcement, which has been developed in Leicester, UK, has previously been described [33]. This procedure was devised based on the observation that the majority of patients referred to our practice were tender over the insertion of the inguinal ligament, lacunar ligament and pectineus muscle. It was postulated that disruption to the inguino-pectineal complex causes chronic pain and treatment should be developed to specifically target this anatomical site. There is evidence that the inguinal and lacunar ligaments are macroscopically disrupted in this group of athletes. Fig. (2) demonstrates a tear in the central part of the inguinal ligament attachment. In a similar fashion to a tenotomy of adductor [34] or forearm extensor tendons [35], it was hypothesised that the release of the inguinal ligament at its point of insertion would alleviate patients' symptoms. The inguinal ligament is divided from the pubic tubercle, releasing the tension at the lacunar ligament. Similarly, if the fascia overlying the pectineus muscle is thickened, the fascia is divided. In these patients the tension in the pectineal ligament is released also. Following the ligamental division, mesh reinforcement is required to prevent herniation and reinforce the anterior abdominal wall. The procedure releases the tension in the area and reduces pressure and tension of the conjoined tendon and posterior inguinal wall.

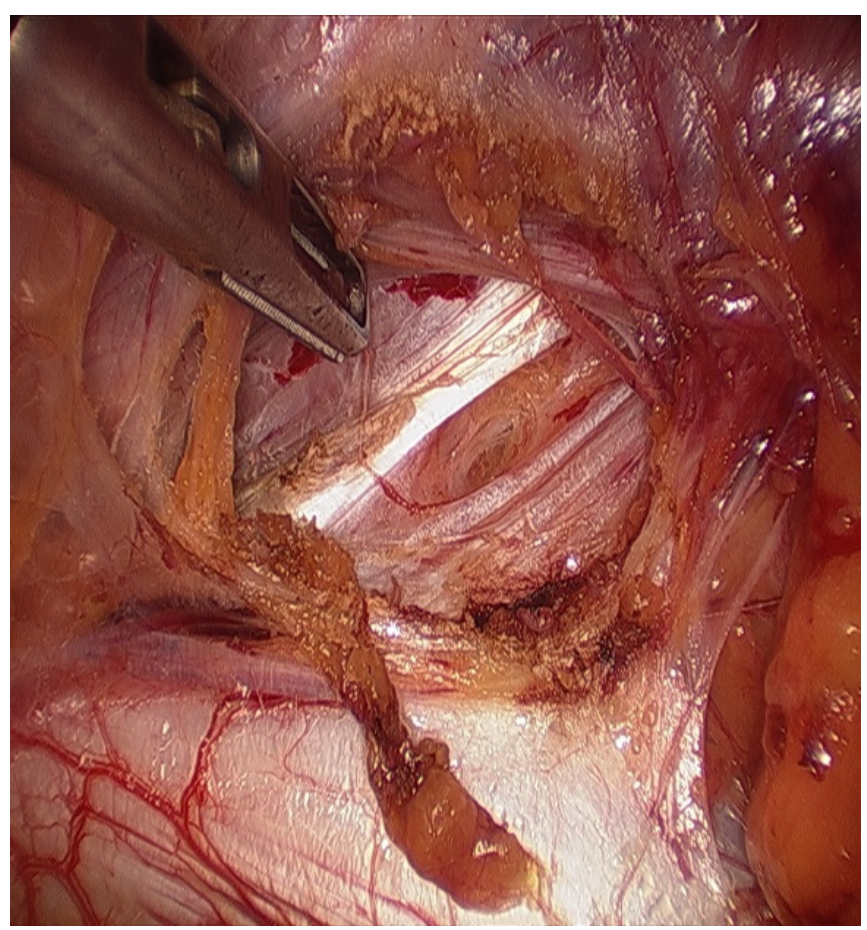

Fig. (2). Photograph demonstrating a tear in the central part of the inguinal ligament attachment.

\subsubsection{Surgical Technique}

The operator and assistant setup is identical to that routinely used for a trans-abdominal pre-peritoneal mesh repair (TAPP) [29]. Pneumo-peritoneum is established using the safe, open technique also developed in Leicester. A small incision is made in the sub-umbilical skin followed by blunt dissection down to the cicatrix. A small incision is made in the cicatrix which is then dilated and a blunt $10 \mathrm{~mm}$ port inserted into the abdomen, through which the telescope is passed. Two other $5 \mathrm{~mm}$ ports are inserted under direct vision. Routine inspection of the hernial orifices is then undertaken. The peritoneum is incised and the whole inguinal region exposed so that the medial end of the inguinal ligament and its surrounding structures can be carefully examined. The femoral canal is also exposed carefully and an assessment made of the attachment of the inguinal, lacunar and pectineal ligaments. The ilio-pubic tract is divided and the inguinal ligament and lacunar ligaments detached from the pubic tubercle using hook diathermy. Excess scar tissue (if present) from previous groin surgery is divided and any old suture material removed. The pectineal ligament, if thickened, is divided also. Following this, a 15 by $12 \mathrm{~cm}$ synthetic mesh is positioned to cover the whole inguinal region and the medial edge secured using a laparoscopic stapler. The peritoneum is then re-attached to prevent any part of the mesh being exposed to the abdominal contents. The three small skin incisions are closed with absorbable vicryl and the wounds infiltrated with local anaesthetic. An ilio-inguinal nerve block using levobupivacaine is performed in all cases. Patients undergo routine post-operative monitoring and are discharged the same day if well.

Return to full activity is encouraged as soon as possible following surgery. Patients are advised to undertake stretching exercises of the groin, incorporating bent knee lunges, and quadriceps stretch of the operated side. Training for sport is strongly encouraged after one week post-surgery and return to regular sporting activity after two weeks.

\subsubsection{Results of the Inguinal Ligament Release Procedure}

To date, more than 400 patients have undergone this procedure now referred to as the Lloyd Release procedure. Our initial published series followed up 48 patients after the procedure, consisting mainly of amateur sportsmen and manual workers [33]. Patients were followed up a median of 6 months after operation using a visual analogue pain score questionnaire to assess the severity of symptoms, frequency of symptoms, and functional limitation. One third of these underwent bilateral procedures. $83.3 \%$ were discharged the same day, with pain control and urinary retention the reasons for overnight stay. Only minor complications were experienced. $92 \%$ of patients returned to normal sporting activity following surgery, with a median return to strenuous activity of 28 days. $76.9 \%$ of patients polled by questionnaire stopped all analgesia post surgery, with the remainder requiring only occasional analgesia. Overall, there was a highly significant improvement in the frequency of pain, severity of pain and functional limitation scores before and after surgery $(\mathrm{p}=0.0012,<0.0001$ and $<0.0001$ respectively). 
Subsequent to this, a prospective study of 73 sportsmen/women was performed which involved a prospective audit and follow-up for least 3 months following surgery [36]. Forty-two were footballers, with 16 professionals, 4 semi-professionals, and 22 amateurs. Twenty-one were rugby players, with 18 professionals and 3 amateurs. The remainder included two professional cricket players, five athletes, one amateur cyclist, one semiprofessional tennis player, and one professional horse rider. Nine rugby players, five footballers and the horse rider had competed at international level. Eleven patients (15\%) had previously had surgery on the symptomatic groin. Previous operations were 6 Gilmore's procedures, 6 inguinal hernia repairs (2 laparoscopic), and 2 adductor tenotomies. The median duration of pain in all patients was 5 months (range 1.5-24 months). Most patients had tried pre-operative physiotherapy with no improvement of symptoms, and $52 \%$ required regular analgesia.
There were no peri-operative complications, or long-term complications such as haematoma, serous formation or wound infection. Post-operatively, patients were mobilising freely at a median of 1 day (range 1-4 days), and were able to perform household chores at a median of 2 days (range 15 days). Returning to light training took a median of 1 week (range 0.5-6 weeks) and full training 3 weeks (range 0.5-12 weeks). Patients had returned to playing sport competitively by a median of 4 weeks post-operatively (range 1-12 weeks), although it should be noted that three operations were performed in the 'off' season. 74\% were able to undertake full training and considered themselves 'match-fit' by 4 weeks. Regular analgesia was required after the first postoperative day in $48 \%$ of patients for a median of 2 days (range 2-28 days).

At follow-up, $73 \%$ of patients reported no symptoms, with the majority of the remainder only reporting mild symptoms. Post-operative pain severity score was a median

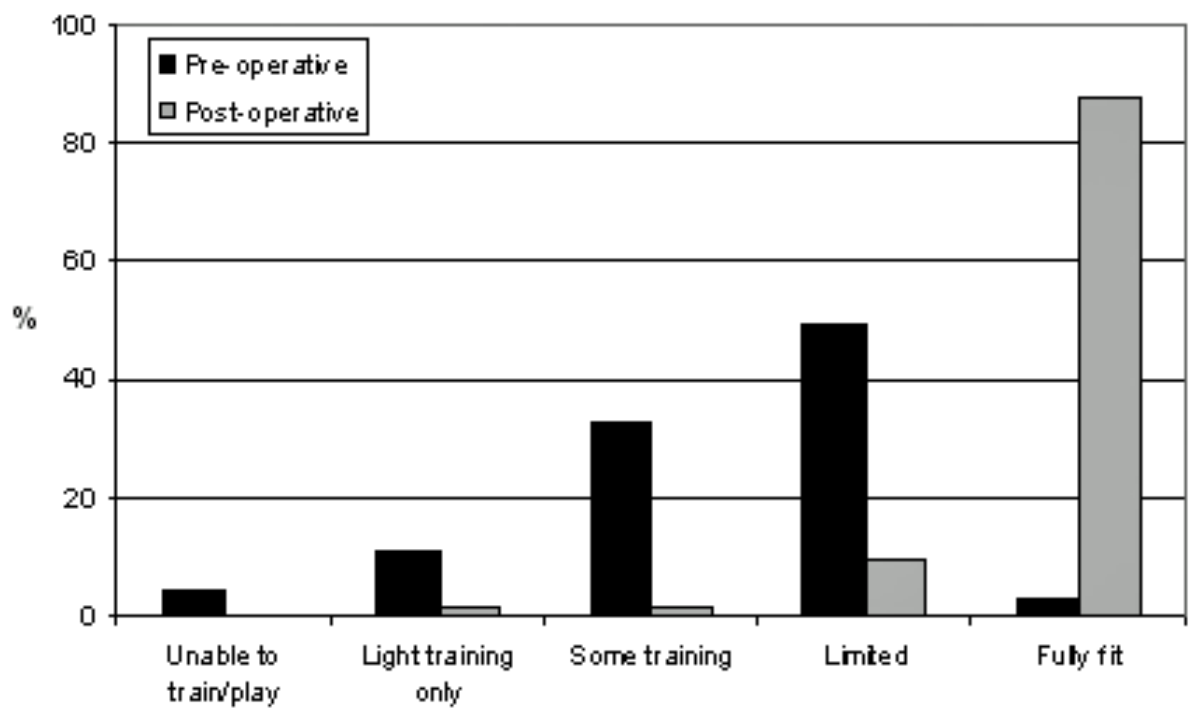

Fig. (3). The Lloyd release Procedure: Functional limitation pre- and post-operatively $(\mathrm{p}<0.001)$. Reproduced with permission of British Journal of Sports Medicine [36].

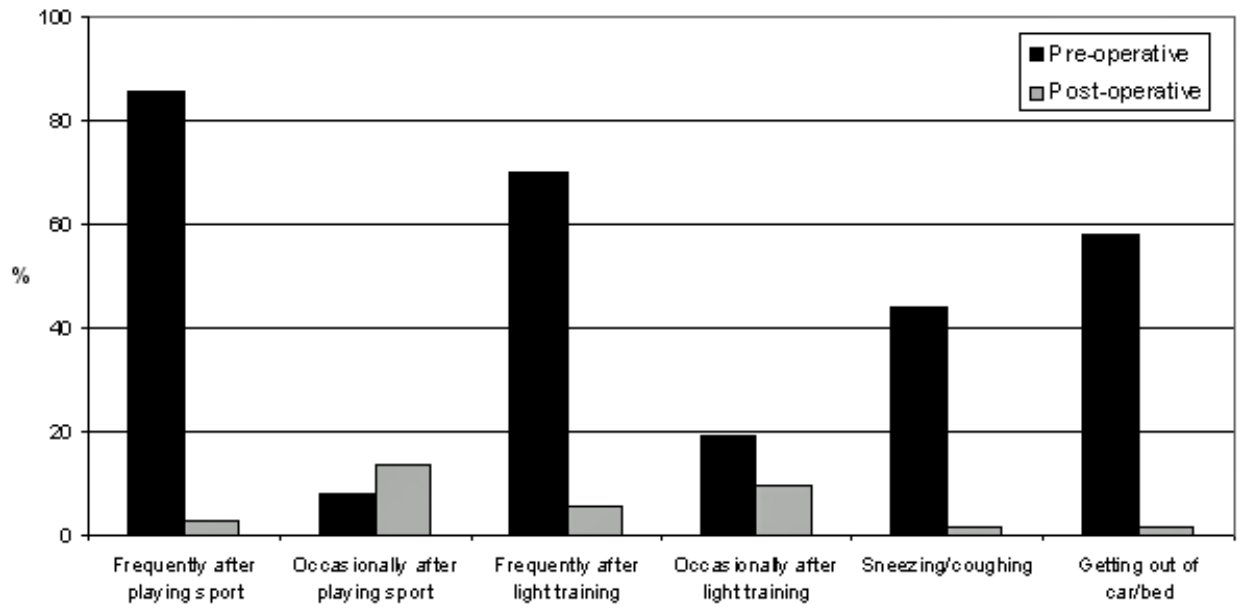

Fig. (4). The Lloyd Release Procedure: Frequency of symptoms in all patients pre-operatively and at follow-up ( $p<0.001$ ). Reproduced with permission of British Journal of Sports Medicine [36]. 


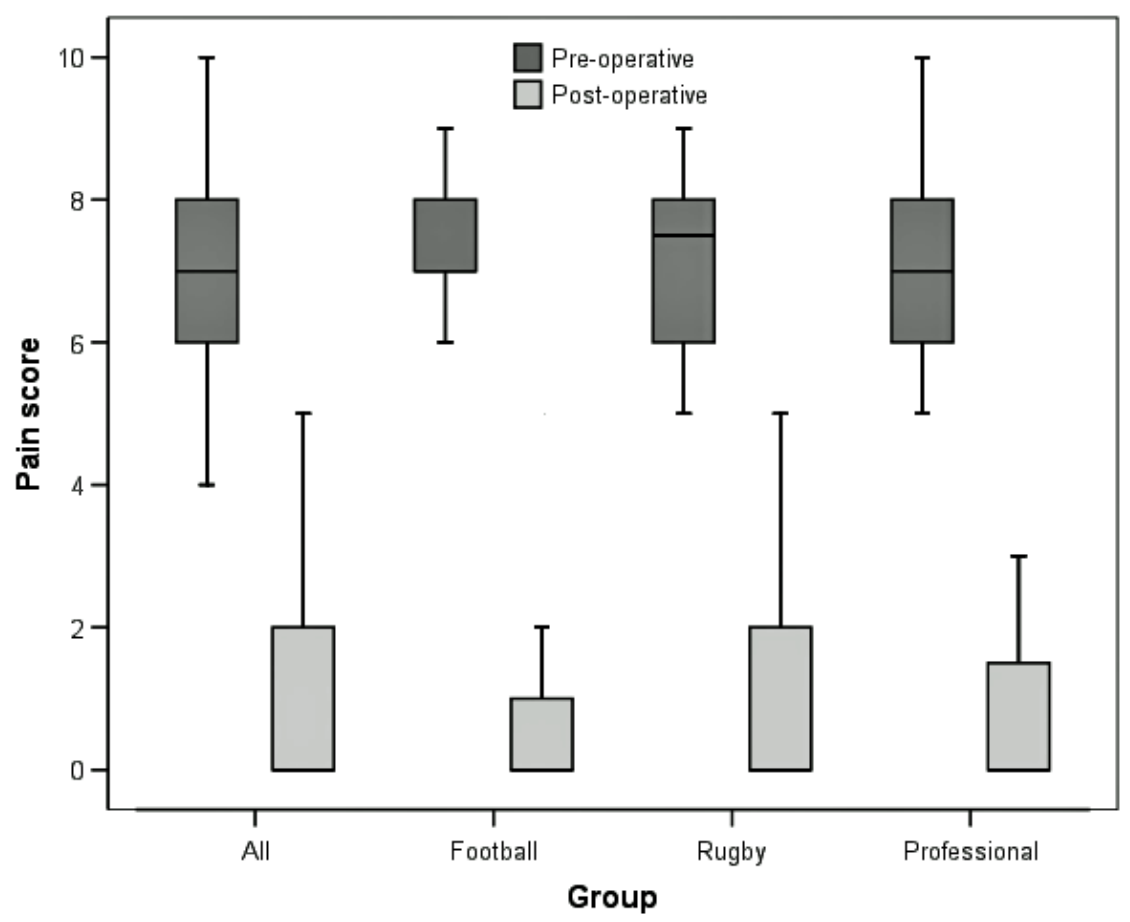

Fig. (5). The Lloyd Release Procedure: Symptom severity score pre- and post-operatively in all patients ( $\mathrm{p}=0.005$ ), football players $(\mathrm{p}<0.001)$, rugby players $(\mathrm{p}<0.001)$, and professional sportsmen $(\mathrm{p}<0.001)$. Reproduced with permission of British Journal of Sports Medicine [36].

of 0 (range $0-8$ ) for the entire group. Two patients reported no change in severity of symptoms, but reduced frequency of occurrence, and two patients reported no change in symptoms following surgery. Only one patient had not returned to playing sport at follow-up. Importantly, no patients reported deterioration in symptoms following surgery. The median change in pain scores between pre- and post-operation was $7(0-10) .88 \%$ of patients had returned to full fitness following the operation. There was a highly significant improvement in frequency of pain, severity of pain, and functional limitation following surgery $(\mathrm{p}<0.001$, $\mathrm{p}=0.005, \mathrm{p}<0.001$ respectively; (Figs. $(\mathbf{3}, \mathbf{4})$ ). Overall, 97\% of patients reported that the operation had improved their symptoms and would recommend the procedure to a colleague/team mate.

When results were sub-analysed with regards to differing sports, both footballers and rugby players achieved good results (Fig. 5). Footballers resumed light training at a median of 2 weeks (range 0.5-6 weeks), full training at 3 weeks (1-12 weeks), and competitive playing at 4 weeks (1.5-12 weeks). $74 \%$ of patients reported no symptoms at follow-up, and $88 \%$ reported return to full fitness. There were highly significant improvements in frequency of pain, severity of pain, and functional limitation following surgery ( $p<0.001$ all cases). Rugby players returned to light training at a median of 1 week (range 0.5-6 weeks), full training at 2 weeks (1-8 weeks), and competitive playing at 4 weeks (2-10 weeks). $76 \%$ of patients reported no symptoms at follow-up, and $90 \%$ reported return to full fitness. There were highly significant improvements in frequency of pain, severity of pain, and functional limitation following surgery $(p<0.001$ all cases).
When professional sportsmen were sub-analysed, light training was resumed at a median of 1 week (range 0.5-6 weeks), full training at 2 weeks (0.5-8 weeks), and competitive playing at 3 weeks (1-10 weeks), however again it should be noted that three players had operations performed in the 'off-season'. 78\% of this group reported no symptoms at follow-up, with only 1 person $(2.7 \%)$ continuing to experience pain frequently after playing sport (Fig. 5). 84\% of the 37 professional sportsmen were able to undertake full training and considered themselves 'match-fit' by 4 weeks. All except one patient (97\%) reported return to full fitness at follow-up, and all of these reported that the operation had improved their symptoms and that they would recommend it to a colleague/team-mate with similar symptoms. There were highly significant improvements in frequency of pain, severity of pain, and functional limitation following surgery $(\mathrm{p}<0.001$ all cases $)$.

\subsubsection{Post-Operative Rehabilitation}

Post-operative rehabilitation programmes vary between published series, although only few detail these comprehensively [22,37]. In general, walking is encouraged immediately following surgery, with jogging and running started by the third week. During this time, physiotherapy to strengthen and increase the flexibility of the hip and abdominal muscles is performed. Cycling can be used to maintain overall conditioning. Sprinting, shuttle runs and kicking exercises are commenced when running can be performed pain-free. Sport-specific training is started at this time, and return to competition when this can be performed pain-free. 


\subsection{Nerve Entrapment Syndromes}

Entrapment or disruption of the ilio-hypogastric, ilioinguinal, genitor-femoral, lateral femoral cutaneous or obturator nerve should all be considered as potential underlying pathology in athletes presenting with groin pain (Fig. 1) [38]. Nerve entrapments are more common following surgical trauma, including hip replacement, however they have been reported following sporting injuries.

\subsubsection{Obturator Nerve}

The obturator nerve arises from the anterior branches of the ventral primary rami of L2-4, which fuse within the psoas muscle and emerge from its medial boarder beneath the common iliac vessels. The nerve then travels along the lateral wall of the lesser pelvis to enter the obturator foramen. It then divides into anterior and posterior branches. The anterior branch passes above obturator externus, supplying pectineus and descends in the thigh deep to adductor longus, which it also supplies. It then passes over adductor brevis, supplying it and gracilis, to end in the subsartorial plexus supplying the skin over the medial side of the thigh. The posterior branch emerges through obturator externus, supplying it, before descending on and supplying adductor magnus.

In athletes, entrapment may occur within the obturator foramen, or more commonly, the posterior branch may become entrapped within adductor magnus [39,40]. The main symptoms including difficulty walking and development of an unstable leg. Groin and medial thigh pain may also occur, which worsen with exercise, particularly after kicking movements. With severe injuries, loss of adduction can occur, with wasting over the adductor muscles and paraesthesia along the medial aspect of the distal thigh. Loss of adductor tendon reflex with preservation of other muscle stretch reflexes often may be observed, as may a positive Howship-Romberg sign (medial knee pain induced by forced hip abduction, extension, and internal rotation). Electromyography of the adductor muscles may be used to confirm the diagnosis, as may nerve blocks $[39,40]$.

Physiotherapy of the adductor muscles may be sufficient for entrapment of the anterior division of the nerve. When obturator nerve denervation is present on electromyography surgery is the primary treatment, dividing the fascia over pectineus and adductor longus and identifying the anterior branch in the space between the two muscles, beneath a thickened fascia, which is divided along the line of the nerve $[39,40]$.

Damage, compression or entrapment of the obturator nerve along its journey through the pelvis, obturator foramen and upper thigh may give rise to groin pain and/or thigh pain. Some authors believe that a primary nerve entrapment syndrome is a common cause of groin pain and that steroid injections into the nerve improve symptoms [40]. It may be that pressure within the pectineus muscle caused by thickening of the pectineus fascia causes compression of the nerve and groin pain. It is our experience that dividing the pectineal fascia also improves the symptoms of upper medial thigh pain and that this could be explained by a possible compartment syndrome within the pectineal fascia. This has not been proven but pressure around or in the pectineal fascia would explain the referred pain to the upper medial thigh experienced by some athletes.

\subsubsection{Ilio-Hypogastric Nerve}

Ilio-hypogastric nerve (T12, L1) - trauma or muscle tears of the lower abdominal wall muscles may result in entrapment of the ilio-hypogastric nerve in scar tissue or neuroma formation [41]. Pain is felt in the supra-pubic and inguinal regions, occasionally extending into the genitalia. Pain can usually be reproduced by palpating over the area of nerve compression. Hypo or hyperaesthesia maybe present in the area supplied by the nerve, although this may be small.

\subsubsection{Ilio-Inguinal Nerve}

Ilio-inguinal nerve $(\mathrm{T} 12, \mathrm{~L} 1)$ - tearing of lower external oblique aponeurosis, particularly in hockey players [42], may result in direct damage to the ilio-inguinal nerve, subsequent compression by scar tissue or neuroma formation. Pain is felt along inguinal ligament, and may radiate into the lower abdominal wall, scrotum/labia, or internal thigh. Hypo or hyperaesthesia maybe present along the internal ligament. Pain can reproduced by palpating over where the nerve exits the inguinal canal, or medial to the anterior superior iliac spine, and symptoms can be increased by hip extension.

\subsubsection{Genito-Femoral Nerve}

Genito-femoral nerve (L1, L2) - genitor-femoral nerve entrapment can occur anywhere along its path on psoas major, the inguinal canal (genital branch), or Sartorius muscle (femoral branch). Entrapment of the femoral branch causes pain and hypo/hyperaesthesia of the anterior thigh below the inguinal ligament, which may increase on hip rotation or prolonged walking.

Diagnosing entrapment of these nerves and distinguishing between them may be difficult and electro-diagnostic investigations are often unhelpful. Diagnosis of iliohypogastric or ilio-inguinal nerve entrapment rest upon history and examination, followed by rapid symptom relief following infiltration of local anaesthetic with or without steroids into the region where the nerves exit the internal oblique. This would have no effect in cases of genitofemoral nerve entrapment, when a block of L1 and L2 nerve roots should result in relief of symptoms. These blocks are both diagnostic and therapeutic. Medical therapy with antiepileptic medications (gabapentin, carbamazepine), as well as tricyclic antidepressents (amitryptiline), topical capsaicin cream and TENS unit may also be of some benefit. Surgical excision [43,44] or ablation [42] is the treatment of choice following failure of conservative management and may be performed endoscopically $[45,46]$.

\subsubsection{Lateral Femoral Cutaneous Nerve}

The lateral femoral cutaneous nerve arises from the ventral primary rami of L2-4. Injury or entrapment results in the syndrome of meralgia paresthetica (derived from the greek meros, meaning thigh, and algo, meaning pain), characterised by paraesthesia and pain in the lateral and anterolateral thigh. Entrapment most commonly occurs as the nerve travels posterior to the inguinal ligament. Patient examination may reveal hyperaesthesia over the lateral thigh and a positive Tinel sign may be present over the inguinal 
ligament, although signs may be absent. Diagnosis may be made using local anaesthetic injection near the inguinal ligament or electrodiagnostic testing. Treatment options include local anaesthetic injection, with or without steroid. NSAIDs, antiepileptic medications, tricyclic antidepressant and capsaicin cream. Patients should avoid further nerve irritation, including avoiding hip extension, prolonged standing and compressive clothing. Surgical nerve decompression or transaction may be performed if conservative management fails [44,47]. The authors' have decompressed the lateral femoral cutaneous nerve by releasing the tight fibrous tunnel through which the nerve travels. This was done laparoscopically with a very good clinical outcome.

\section{OSTEITIS PUBIS}

Osteitis pubis is a chronic non-infectious inflammatory condition involving the pubic symphysis, resulting in resorption of the bone or cartilage of the pubic symphysis due to repetitive stress $[48,49]$. It commonly presents with insidious onset of pain in the adductor region and around the pubic symphysis. Osteitis pubis is particularly common in sports such as football, ice hockey and rugby - activities which involve sprinting, kicking and rapid change of direction, and it is thought to be secondary to shearing or rotational movement of the pubic symphysis [50-52]. It may also be associated with pregnancy, trauma, rheumatological disease and gynaecological/urological surgery. Examination reveals tenderness to palpation of the pubic tubercle, with pain increasing on resisted thigh adduction and contraction of rectus abdominis. X-ray examination demonstrates subchondral erosions, sclerosis, and widening of the symphysis (Fig. 6). Early MRI changes include subchondral bone marrow oedema, fluid in symphysis pubis joint, and periarticular oedema [53]. Chronic changes ( $>6$ months) include subchondral sclerosis, subchondral resorption and bony margin irregularities, and osteophytes formation [53].

A recent systematic review has examined the treatment of osteitis pubis in athletes [54]. They only identified level 4 evidence in the literature - 25 case series/reports of osteitis pubis treatment reporting outcome in 195 athletes. No randomised controlled trials have been conducted. Based upon this evidence, very few conclusions can be drawn as to the best treatment of these patients. The majority of cases are self-limiting; therefore conservative treatment is typically the first-line treatment - rest, non-steroidal anti-inflammatory drugs (NSAIDs), heat treatment, compression shorts, and rehabilitative physiotherapy, particularly focusing on core muscle stability. Average return to sporting activity was 9.5 weeks in these patients, with 41 of 42 patients returning to full activity [51, 55-59].

Some series advocated intra-symphysial corticosteroid injection, with or without local anaesthetic, however this remains controversial because of concerns regarding symphysis loosening. Three case series exist in the literature, each differing in type, dose and strength of medication, thus limiting conclusions [60-62]. Whilst the average return to sporting activity for 'responders' was 8 weeks, it should be noted that $20.7 \%$ were unable to return to any sporting activity. A greater response was seen in those patients treated earlier in the course of the disease.
Proliferative Injection Therapy (Prolotherapy) involves injecting an otherwise non-pharmacological and non-active irritant solution into the body, for the purpose of strengthening weakened connective tissue of tendons and ligaments to alleviating pain. Prolotherapy using dextrose and lignocaine has been used to treat osteitis pubis in a series of 24 athletes [63]. Multiple injections sites across the pubic rami and symphysis for an average of 2.8 treatments resulted in a high treatment success rate, with $91.7 \%$ of athletes returning to sport after an average of 9 weeks, with zero complications. Clearly this is a therapeutic modality worthy of further consideration.

As mentioned, most cases of osteitis pubis are selflimiting after a period of 2-3 months, although some authors report the average healing time to be 9-10 months. Surgical intervention is reserved for symptoms refractory to conservative management. Options include curettage of the pubic symphysis [64, 65], polypropylene mesh placement into the preperitoneal retropubic space $[66,67]$, and pubic symphysis stabilisation $[68,69]$. Only six case series exist in the literature, reporting 46 athletes. Thirty-seven returned to playing sport after an average of 22.4 weeks following curettage (18 of 25 patients) [64, 65], 7.2 weeks following polypropylene mesh placement (12 of 13 patients) [66, 67], and 26.4 weeks following pelvic stabilisation (7 of 8 patients) $[68,69]$. Only 2 minor complications were reported $(4.3 \%)$. No study compared surgical technique, therefore comparison is not possible.

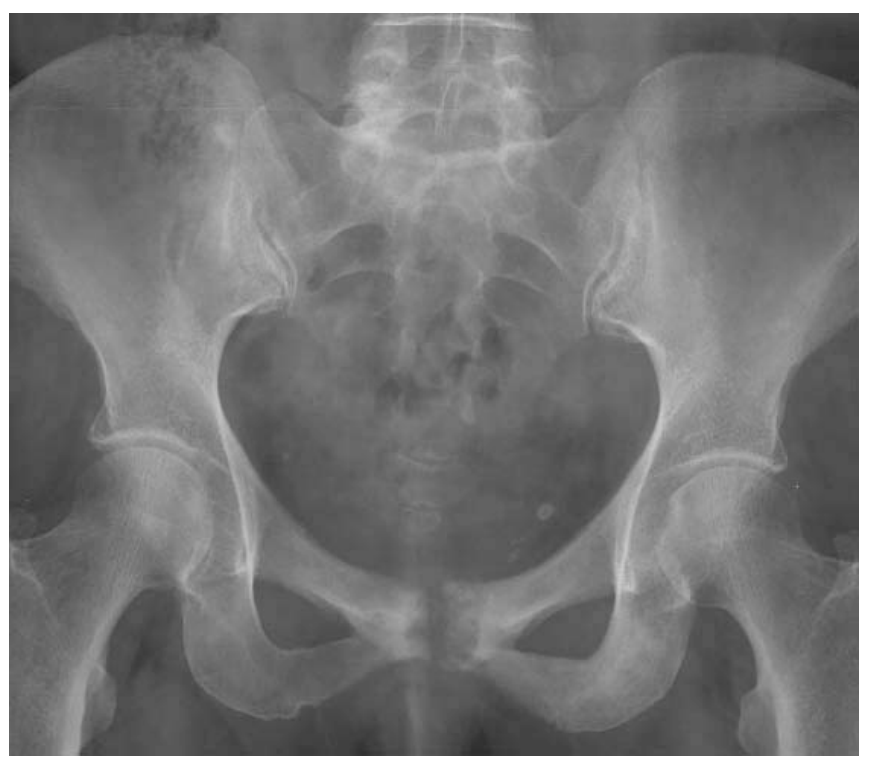

Fig. (6). Plain radiograph in a patient with osteitis pubis demonstrating erosive change, joint irregularity and sclerosis of the pubic symphysis (Image courtesy of Dr Donna D'Souza and Radiopaedia.org).

\section{ADDUCTOR MUSCLE DISORDERS}

An adductor strain is a common injury, particularly in those participating in sports such as football, hockey, martial arts and tennis. Injuries most commonly occur forceful abduction of the thigh occurs during an intentional adduction, for example when a footballer attempts to kick a ball and meets resistance from a tackling opposing player. Acute adductor strains most commonly occurs at the musculotendinous junction, with the athlete complaining of 
pain proximally at the site of the adductor longus tendon, exacerbated by adduction of the thigh. The adductor tendons have small insertions that attach to periosteum-free bone, which have poor blood supplies and rich innervations, resulting in high levels of pain and poor healing capacity. Injury is associated with failure to stretch the adductor muscles properly prior to exercise and also adductor muscle weakness. Weakness of the hip adductors compared to hip abductors pre-season has been found to be a risk factor for development of groin injury in ice hockey players [70], and development of a pre-season exercise program resulting in a significant reduction in hip adductor injuries [71].

Acute injuries are often noticed as a sudden stabbing pain in the groin. Muscle function tends to be preserved unless a grade 3 tear is present, in which loss of adduction occurs. In cases of loss of muscle function, obturator nerve entrapment should be considered. Clinical signs include tenderness, swelling and bruising of the superior medial thigh with pain on resisted adduction and passive abduction of the hip. Occasionally a defect in the adductor muscles can be palpated.

Inadequate management of acute strains or premature return to sporting activities can result in chronic strains. Chronic adductor strains tend to cause a dull ache in the groin, which may radiate down the medial aspect of the thigh or proximally into rectus abdominis. This may be associated with stiffness in the groin, particularly in the morning or at the beginning of exercise. Pain and stiffness may improve with activity, but recur after activity. On examination, patients may be tender over the inferior pubic ramus at the origins of adductor longus and gracilis, which pain worsening on resisted adduction.

\subsection{Treatment}

There is very little evidence base for adductor injuries, with very few comparative trials reported. All acute injuries are initially managed with rest, ice, compression and elevation. Ultrasound scan or MRI may be used to evaluate the degree of injury. For grade I and grade II injuries passive range of motion physiotherapy should begin immediately. Stretching and progressive strengthening exercises should be performed as soon as can be performed pain-free, which can include a slide board and lateral lunges. Grade III injuries ( $>90 \%$ muscle fibres torn) should initially be treated with rest and compression. Passive range of movement exercises should be started after day 3 and crutches should be used until pain-free ambulation can be performed. After one week, pain-free stretching exercises can be performed with progressive resistance strengthening exercises. Slide board exercises, progressing to sport-specific exercises can be performed after 10-14 days. Tendon repair surgery is only indicated in acute grade III strains when there is complete rupture [72], although a recent series of 19 American Football players with acute complete proximal adductor longus tendon rupture demonstrated that conservative management with a staged physiotherapy programme resulted in faster return to full sporting activity (mean 6.1 vs 12.0 weeks) [73].

Failure to adequately treat acute injuries or to leave sufficient time for healing may lead to chronic strains. In the treatment of chronic strains, rest, ice, massage, and therapeutic ultrasound have been recommended. NSAIDs have been suggested, but no controlled trials have been published, and local anaesthetic/steroid injections have been shown to result in only short term relief of symptoms [74]. A careful monitored rehabilitation program with a total cessation of the sports activity is necessary for the chronic adductor injury to heal and become pain-free. This should consist of isometric exercises, strengthening of the hip- and pelvis-stabilizing muscles, and proprioceptive training. No increase in pain should be experienced during or after the exercises. The load of the exercises gradually is increased. Specific strengthening of the adductor muscles then is implemented. Cycling and swimming are useful to maintain general fitness. Running is only commenced after the above activities can be performed pain-free at high intensity. When running is pain-free, sprinting and shuttle-runs can then be started. Sport-specific activities are the final step in rehabilitation prior to full return to sport. Rehabilitation is often prolonged, taking up to 6 months or more. Prolotherapy may have a role in the treatment of chronic adductor injuries. Topol et al. [75] reported a series of 75 elite athletes with chronic groin pain of a mean duration of 11 months. They received monthly injections of $12.5 \%$ dextrose with $0.5 \%$ lignocaine into the adductor attachments to the pubis. After an average of 3 treatments, 69 (92\%) reported an improvement in symptoms, with $82 \%$ improvement in visual analog score of pain in sport and 78\% reduction in the Nirschl pain score. Return to unrestricted sport occurred after an average of 3 months.

Surgical intervention is indicated in chronic strains refractory to conservative treatment. Martens et al. [76] performed adductor tenotomy in combination with rectus abdominis tenotomy in a series of 81 athletes. A compression bandage was applied for 24 hours following surgery, the patient walking after 2 days and resumed running within pain limits 5 weeks postoperatively. The time to return to full sporting activities was 12 weeks. In this study, there was no loss of power in the surgical group compared with the control group. Akermark et al. [34] reported a series of 16 adductor longus tendon tenotomies in athletes with long standing groin pain and focal tenderness over the adductor origin, refractory to conservative treatment. At a mean follow up of 35 months after surgery, all patients were improved or free of symptoms. All but 1 of the athletes returned to the same sport within a mean of 6.6 weeks, and 12 of 16 returned to competitive sports within a mean of 14 weeks after surgery. A majority of the patients (10 of 16) returned to full athletic activity, whereas 5 of 16 performed at a reduced level. One patient discontinued his sports activity due to other causes. Van der Donckt et al. [77] reported a series of 41 athletes with chronic groin pain resistant to conservative treatment treated with a Bassini's hernia repair and percutaneous adductor longus tenotomy. All patients had resumed their sporting activities on average 6.9 months after surgery (range 6 to 15 months). Thirtyseven performed at the same level and 4 at a reduced level. Only one patient performed at a lower level due to persistent groin pain. Although these data suggest that adductor tenotomy may be of benefit in chronic injury, refractory to conservative treatment, there is a need for randomised trials to evaluate surgery against prolotherapy and physiotherapy.

\section{DISCUSSION}

There is no consensus in the literature concerning the surgical management of athletes with sportsman' groin pain. 
Regardless of approach, laparoscopic or open, results vary considerably in the literature. The results of the inguinal release procedure compare favourably with results in the literature - overall $97 \%$ of patients in this cohort reported improvement in symptoms with the procedure, with $99 \%$ having returned to sporting activity. The literature reports rates of $63-100 \%$ returning to sporting activity following surgical intervention. Our patients returned to playing competitively a median of 4 weeks following surgery. This is comparable with the best results achieved in the literature. It is evident from the literature that laparoscopic hernia repair alone does not benefit all patients with chronic groin pain, however it remains to be established if the addition of an inguinal tenotomy is beneficial, or if the results observed solely due to mesh reinforcement. A randomised-controlled trial of laparoscopic hernia repair versus hernia repair and inguinal ligament release would be necessary to resolve this.

Laparoscopic hernia surgery has been shown to be associated with short hospital stay, improved post-operative pain, and improved convalescence. Several series have explored the benefits of laparoscopic hernia repair as a treatment modality in athletes with chronic groin pain [2228]. Post-operative results range from $100 \%$ returning to sporting activities at 4 weeks, to $93 \%$ within 3 months. In the Leicester series, $74 \%$ had returned to 'match-fitness' within 4 weeks of operation. The series of 73 patients is larger than most previously reported laparoscopic series; four of these series report cohorts of 15 patients or less [23, 25, 27, 28]. Laparoscopic surgery allows direct visualisation of the entire posterior wall of both inguinal canal, thus enabling bilateral reinforcement from pubis to anterior superior iliac spine [26]. Laparoscopic repair seems to achieve faster rehabilitation and return to sporting activities than open repair, with the majority having returned to full sporting activity between 4 and 12 weeks. It remains to be seen whether laparoscopic or open repair is more durable - despite being not directly comparable to sportsman's groin, a Cochrane review found no difference in hernia recurrence between open and laparoscopic repair [78].

Several aspects make comparisons between series difficult. Differing mixes of sports (i.e. football, rugby, Australian rules football etc.) and heterogeneous populations between studies, and variations in definitions used hamper reporting and make comparing results between series unreliable. Some studies use return to competitive level, others to any sporting activity. This is an important consideration when interpreting data between other published reports. In addition, basing surgical success on return to sports alone is inadequate; standardised and validated reporting criteria need to be developed.

With increasing surveillance and recognition, along with modern trends in regular exercise, the number of athletes, both professional and recreational, presenting with 'sportsman's groin' will increase. It remains a poorly defined and researched condition and it is still not known which operative approach is optimal for these patients. Well-designed trials are necessary to compare and evaluate treatment modalities, improve postsurgery rehabilitation, and to develop preventative conditioning programmes to reduce the frequency of this injury.

\section{REFERENCES}

[1] Lovell G. The diagnosis of chronic groin pain in athletes: a review of 189 cases. Aust J Sci Med Sport 1995; 27: 76-9.
[2] Gilmore J. Groin pain in the soccer athlete: fact, fiction, and treatment. Clin Sports Med 1998; 17(4): 787-9.

[3] Walden M, Hagglund M, Ekstrand J. UEFA Champions League study: a prospective study of injuries in professional football during the 20012002 season. Br J Sports Med 2005; 39(8): 542-6.

[4] Hackney RG. The sports hernia: a cause of chronic groin pain. Brit J Sport Med 1993; 27(1): 58-62.

[5] Kemp BS. The 'sports hernia': a common cause of groin pain. Physician Sports Med 1998; 26: 36-44.

[6] Orchard JW, Read JW, Neophyton J, Garlick D. Groin pain associated with ultrasound finding of inguinal canal posterior wall deficiency in Australian Rules footballers. Brit J Sport Med 1998; 32: 134-9.

[7] Meyers WC, Foley DP, Garrett WE, Lohnes JH, Mandlebaum B. Management of severe lower abdominal or inguinal pain in highperformance athletes. Am J Sports Med 2000; 28: 2-8.

[8] Gilmore J. Groin pain in the soccer athlete: fact, fiction and treatment. Am J Sports Med 1998; 32: 1238-42.

[9] Verrall GM, Slavotinek JP, Barnes PG, et al. Hip joint range of motion restriction precedes athletic chronic groin injury. J Sci Med Sport 2007; 10: 463-6.

[10] Biedert RM, Warnke K, Meyer S. Symphysis syndrome in athletes. surgical treatment for chronic lower abdominal, groin, and adductor pain in athletes. Clin J Sport Med 2003; 13(5): 278-4

[11] De Paulis F, Cacchio A, Michelini O, et al. Sports injuries in the pelvis and hip: Diagnostic imaging. Eur J Radiol 1998; 27: S49-S59.

[12] Orchard JW, Read JW, Neophyton J, et al. Groin pain associated with ultrasound finding of inguinal canal posterior wall deficiency in Australian Rules footballers. Br J Sports Med 1998; 32: 134-9.

[13] LeBlanc KE, LeBlanc KA. Groin pain in athletes. Hernia 2003; 7: 6871.

[14] Malycha P, Lovell G. Inguinal surgery in athletes with chronic groin pain: the 'sportsman's' hernia. ANZ J Surg 1992; 62(2): 123-5.

[15] Steele P, Annear P, Grove JR. Surgery for posterior inguinal wall deficiency in athletes. J Sci Med Sport 2004; 7(4): 415-21.

[16] Canonico S, Bebvento R, Della-Corte A, Fattopace A, Canonico R. Sutureless tension-free hernia repair with human fibrin glue (tissucol) in soccer players with chronic inguinal pain: initial experience. Int J Sports Med 2007; 28(10): 873-6.

[17] Taylor DC, Meyers WC, Moylan JA et al. Abdominal musculature abnormalities as a cause of groin pain in athletes. Inguinal hernias and pubalgia. Am J Sports Med 1991; 19(3): 239-42.

[18] Simonet WT, Saylor HL, Sim L. Abdominal wall muscle tears in hockey players. Int J Sports Med 1995; 16(2): 126-8.

[19] Brannigan AE, Kerin MJ, McEntee GP. Gilmore's groin repair in athletes. J Orthop Sports Phys Ther 2000; 30(6): 329-32.

[20] Van der Donckt K, Steenbrugge F, Abbeele K, Verdonk R, Verhelst M. Bassini's hernial repair and adductor longus tenotomy in the treatment of chronic groin pain in athletes. Acta Orthop Belg 2003; 69(1): 35-41.

[21] Polglase AL, Frydman GM, Farmer KC. Inguinal surgery for debilitating chronic groin pain in athletes. Med J Aust 1991; 155(10): 674-7.

[22] Veen RN, Baat P, Heijboer MP, et al. Successful endoscopic treatment of chronic groin pain in athletes. Surg Endosc 2007; 21: 189-93.

[23] Srinivasan A, Schuricht A. Inguinal surgery in athletes with chronic groin pain: the 'sportsman's' hernia. J Laparoendosc Adv Surg Tech A 2002; 12(2): 101-6.

[24] Paajanen H, Syvahuoko I, Airo I. Totally extraperitoneal endoscopic (TEP) treatment of sportsman's hernia. Surg Laparosc Endos Percutan Tech 2004; 14(4): 215-8.

[25] Kluin J, Hoed PT, Linschoten R, Ijzerman JC, Steensel CJ. Endoscopic evaluation and treatment of groin pain in the athlete. Am J Sports Med 2004; 32: 944-9.

[26] Genitsaris M, Goulimaris I, Sikas N. Laparoscopic repair of groin pain in athletes. Am J Sports Med 2004; 32(5): 1238-42.

[27] Azurin DJ, Go LS, Schuricht A, McShane J, Bartolozzi A. Endoscopic preperitoneal herniorrhaphy in professional athletes with groin pain. J Laparoendosc Adv Surg Tech A 1997; 7(1): 7-12.

[28] Edelman DS, Selesnick H. 'Sports' hernia: treatment with biologic mesh (Surgisis). Surg Endosc 2006; 20: 971-3.

[29] Memon M, Fitzgibbons R. Laparoscopic Inguinal Hernia Repair: Transabdominal Preperitoneal (TAPP) and Totally Extraperitoneal (TEP). The SAGES Manual, Chapter 40, Second Edition, Springer New York 1999.

[30] Brooks DC. A prospective comparison of laparoscopic and tension-free open herniorrhaphy. Arch Surg 1994; 129: 361-6. 
[31] Kozol R, Lange PM, Kosir M, et al. A prospective randomized study of open $v$ laparoscopic inguinal hernia repair. Arch Surg 1997; 132: 2925.

[32] Liem MS, Graaf YV, Steensel CJ, et al. Comparison of conventional anterior surgery and laparoscopic surgery for inguinal hernia repair. $\mathrm{N}$ Engl J Med 1997; 336: 1541-7.

[33] Lloyd DM, Sutton CD, Altafa A, et al. Laparoscopic inguinal ligament tenotomy and mesh reinforcement of the anterior abdominal wall: a new approach for the management of chronic groin pain. Surg Endosc Laparosc Percut Tech 2008; 18(4): 363-8.

[34] Akermark C, Johansson C. Tenotomy of the adductor longus tendon in the treatment of chronic groin pain in athletes. Am J Sports Med 1992; 20(6):640-3.

[35] Sverniov B, Adolfsson L. Outcome of release of the lateral extensor muscle origin for epicondylitis. Scand J Plast Reconstr Hand Surg 2006; 40: 161-5.

[36] Mann CD, Sutton CD, Garcea G, Lloyd DM. The inguinal release procedure for groin pain: initial experience in 73 sportsmen/women. $\mathrm{Br}$ J Sports Med 2009; 43(8): 579-83.

[37] Hemingway AE, Herrington L, Blower AL. Changes in muscle strength and pain in response to surgical repair of posterior abdominal wall disruption followed by rehabilitation. Br J Sports Med 2003; 37:54-8.

[38] Akita K, Niga S, Yamato Y, Muneta T, Sato T. Anatomic basis of chronic groin pain with special reference to sports hernia. Surg Radiol Anat 1999; 21(1): 1-5.

[39] Tipton JS. Obturator neuropathy. Curr Rev Musculoskelet Med 2008; 1(3-4): 234-7.

[40] Bradshaw C, McCrory P, Bell S, Brukner P. Obturator nerve entrapment. A cause of groin pain in athletes. Am J Sports Med 1997; 25(3): 402-8.

[41] Ziprin P, Williams P, Foster ME. External oblique aponeurosis nerve entrapment as a cause of groin pain in the athlete. Br J Surg 1999; 86(4): 566-8

[42] Irshad K, Feldman LS, Lavoie C, Lacroix VJ, Mulder DS, Brown RA. Operative management of Hockey groin syndrome: 12 years of experience in National Hockey League Players. Surgery 2001; 130(4): 759-66.

[43] Brown RA, Mascia A, Kinnear DG, Lacroix V, Feldman L, Mulder DS. An 18-year review of sports groin injuries in the elite hockey player: clinical presentation, new diagnostic imaging, treatment, and results. Clin J Sport Med 2008; 18(3): 221-6.

[44] Lee CH, Dellon AL. Surgical management of groin pain of neural origin. J Am Coll Surg 2000; 191(2): 137-42.

[45] Choller S. Retroperitoneal endoscopic neurectomy for nerve entrapment after hernia repair. Br J Surg 1997; 84(2): 216-9.

[46] Giger U, Wente MN, Büchler MW, Krähenbühl S, Lerut J, Krähenbühl L. Endoscopic retroperitoneal neurectomy for chronic pain after groin surgery. Br J Surg 2009; 96(9): 1076-81.

[47] Khalil N, Nicotra A, Rakowicz W. Treatment for meralgia paraesthetica. Cochrane Database Syst Rev 2008; (3): CD004159.

[48] Batt M, McShane J, Dillingham M, et al. Osteitis pubis in collegiate football players. Med Sci Sports Exerc 1995; 27: 629-33.

[49] Fricker PA, Taunton JE, Ammann W. Osteitis pubis in athletes: infection, inflammation, or injury? Sports Med 1991; 12: 266-79.

[50] Holt M, Keene J, Graf B, et al. Treatment of osteitis pubis in athletes: Results of Corticosteroid Injections. Am J Sports Med 1995; 23: 601-6

[51] McKim K, Taunton J. The Effectiveness of Compression Shorts in the Treatment of Athletes with Osteitis pubis N Z J Sports Med 2001; 29: 70-3.

[52] Verrall GM, Slavotinek JP, Fon GT. Incidence of pubic bone marrow oedema in Australian rules football players: relation to groin pain. $\mathrm{Br} \mathrm{J}$ Sports Med 2001; 35: 28-33.

[53] Kunduracioglu B, Yilmaz C, Yorubulut M, Kudas S. Magnetic resonance findings of osteitis pubis. J Magn Reson Imaging 2007; 25 : 535-9

[54] Choi H, McCartney M, Best TM. Treatment of osteitis pubis and osteomyelitis of the pubic symphysis in athletes: a systematic review. Br J Sports Med 2009. [Epub ahead of print].
[55] McMurtry C, Avioli L. Osteitis pubis in athlete. Calcif Tissue Int 1986; 38:76-7.

[56] McCarthy A, Vincenzino B. Treatment of osteitis pubis via the pelvic muscles. Man Ther 2003; 8: 257-60.

[57] Rodriguez C, Miguel A, Lima H, et al. Ostetis pubis syndrome in the professional soccer athlete: a case report. J Athl Train 2001; 36: 437-40.

[58] Sing R, Coredes R, Siberski D. Osteitis pubis in the active patient. Phys Sports Med 1995; 23: 67-73.

[59] Wollin M, Lovell G. Osteitis pubis in four young football players: a case series demonstrating successful rehabilitation. Phys Ther Sport 2006; 7: 153-60.

[60] Holt M, Keene J, Graf B, et al. Treatment of osteitis pubis in athletes: Results of Corticosteroid Injections. Am J Sports Med 1995; 23: 601-6

[61] O’Connell M, Powell T, McCaffrey N, et al. Symphyseal cleft injection in the diagnosis and treatment of osteitis pubis in athletes. AJR Am J Roentgenol 2002; 179: 955-9.

[62] Batt M, McShane J, Dillingham M, et al. Osteitis pubis in collegiate football players. Med Sci Sports Exerc 1995; 27: 629-33.

[63] Topol G, Reeves K, Hassanein K. Efficacy of dextrose prolotherapy in elite male kicking-sport athletes with chronic groin pain. Arch Phys Med Rehab 2005; 86: 697-702.

[64] Radic R, Annear P. Use of pubic symphysis curettage for treatmentresistant osteitis pubis in athletes. Am J Sports Med 2008; 36(1): 122-8.

[65] Mulhall K, Mckenna J, Walsh A, et al. Osteitis Pubis in professional soccer players: a report of outcome with symphyseal curettage in cases refractory to conservative management. Clin J Sport Med 2002; 12: 179-81.

[66] Paajanen H, Heikkinen J, Hermunen H, et al. Succesfull treatment of osteitis pubis by using totally extraperitoneal endoscopic technique. Int J Sports Med 2005; 26: 303-6.

[67] Paajanen H, Hermunen H, Karonen J, et al. Pubic magnetic resonance imaging findings in surgically and conservatively treated athletes with osteitis pubis compared to asymptomatic athletes during heavy training. Am J Sports Med 2008; 36(1): 117-21.

[68] Vitanzo P, McShane J. Osteitis pubis. Phys Sports Med 2001; 29(77): 33-8.

[69] Williams P, Thomas D, Downes E. Osteitis pubis and instability of the pubic symphysis when nonoperative measures fail. Am J Sports Med 2000; $28: 350-5$.

[70] Tyler TF, Nicholas SJ, Campbell RJ, et al. The association of hip strength and flexibility with the incidence of adductor muscle strains in professional ice hockey players. Am J Sports Med 2001; 29: 124-8.

[71] Tyler TF, Nicholas SJ, Campbell RJ, et al. The effectiveness of a preseason exercise program on prevent adductor muscle strains in professional ice hockey players. Am J Sports Med 2002; 30: 680-3.

[72] Rizio L, Salvo JP, Schurhoff MR, Uribe JW. Adductor longus rupture in professional football players: acute repair with suture anchors. A report of two cases. Am J Sports Med 2004; 32(1): 243-5.

[73] Schlegel TF, Bushnell BD, Godfrey J, Boublik M. Success of nonoperative management of adductor longus tendon ruptures in National Football League athletes. Am J Sports Med 2009; 37(7): 13949.

[74] Schilders E, Bismil Q, Robinson P, O'Connor PJ, Gibbon WW, Talbot JC. Adductor-related groin pain in competitive athletes. Role of adductor enthesis, magnetic resonance imaging, and entheseal pubic cleft injections. J Bone Joint Surg Am 2007; 89(10): 2173-8.

[75] Topal GA, Reeves KD. Regenerative injection of elite athletes with career-altering chronic groin pain who fail conservative treatment: a consecutive series. Am J Phys Med Rehabil 2008; 87(11): 890-902.

[76] Martens MA, Hansen L, Mulier JC. Adductor tendinitis and musculus rectus abdominis tendopathy. Am J Sports Med 1987; 15(4): 353-6.

[77] Van Der Donckt K, Steenbrugge F, Van Der Abbeele K, Verdonk R, Verhelst M. Bassini's hernia repair and adductor longus tenotomy in the treatment of chronic groin pain in athletes. Acta Orthop Belg 2003; 69(1): 35-41.

[78] McCormack K, Scott N, Go PM, Ross SJ, Grant A. Laparoscopic techniques versus open techniques for inguinal hernia repair. Cochrane Database Syst Rev 2003; 1: CD001785. 